СЛУЧАЙ ИНСУЛИНОМЫ, ВЫЯВЛЕННОЙ У ПАЦИЕНТА ПОСЛЕ БАРИАТРИЧЕСКОЙ ОПЕРАЦИИ ПО ПОВОДУ МОРБИДНОГО ОЖИРЕНИЯ

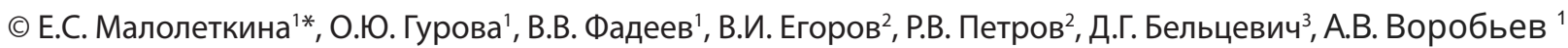

'ФГАОУ ВО Первый МГМУ им. И.М. Сеченова Минздрава России, Москва, Россия

2Городская клиническая больница №5 Департамента здравоохранения г. Москвы, Россия

${ }^{3}$ ФГБУ «Национальный медицинский исследовательский центр эндокринологии» Минздрава России, Москва, Россия

Распространенность инсулином составляет 1-3 случая на один миллион населения в год, из которых в 4-14\% случаев опухоль является злокачественной. Увеличение массы тела является одним из проявлений заболевания, нередко приводя к морбидному ожирению с показаниями для оперативного лечения. Представленный клинический случай, демонстрирует успешное лечение пациента с низким функциональным статусом на фоне злокачественной инсулиномы с манифестацией рефрактерного гипогликемического синдрома после проведения билиопанкреатического шунтирования с продольной резекцией желудка по поводу морбидного ожирения.

КЛЮЧЕВЫЕ СЛОВА: гипогликемический синдром, злокачественная инсулинома, морбидное ожирение, бариатрическая хирургия, билиопанкреатическое шунтирование, клинический случай.

\title{
CASE OF INSULINOMA DETECTED IN A PATIENT AFTER BARIATRIC OPERATION FOR MORBID OBESITY
}

(c) Ekaterina. S. Maloletkina ${ }^{1 *}$, Olesya. Y. Gurova', Valentin V. Fadeyev' ${ }^{1}$ Vyacheslav I. Egorov², Roman V. Petrov², Dmitriy G. Beltsevich ${ }^{3}$, Andrei. V. Vorob'ev ${ }^{1}$

\author{
${ }^{1}$ I.M. Sechenov First Moscow State Medical University (Sechenov University), Moscow, Russia \\ ${ }^{2}$ Moscow city hospital № 5, Moscow, Russia \\ ${ }^{3}$ Endocrinology Research Center, Moscow, Russia
}

The prevalence of insulinomas is $1-3$ cases per million population per year of which $4-14 \%$ tumor is malignant. Weight gain is one of the symptoms of the disease, often resulting in morbid obesity with indications for surgical treatment. The presented clinical case demonstrates the successful treatment of malignant insulinoma with the manifestation of hypoglycemic syndrome after carrying out biliopancreatic bypass with longitudinal gastrectomy for morbid obesity.

KEYWORDS: hypoglycemic syndrome, malignant insulinoma, morbid obesity, bariatric surgery, biliopancreatic diversion, case report.

\section{ВВЕДЕНИЕ}

Инсулинома - нейроэндокринная опухоль, секретирующая избыточное количество инсулина, что приводит к развитию гипогликемического синдрома.

Распространенность инсулином составляет 1-3 случая на один миллион населения в год, из которых в 4-14\% случаев опухоль является злокачественной [1, 2].

От момента появления первых адренергических и нейрогликопенических симптомов до верификации диагноза может проходить от одного месяца до 17 лет. Увеличение массы тела является одним из проявлений инсулином, нередко приводя к морбидному ожирению с показаниями для хирургического лечения в виду неэффективности консервативной терапии. [1].

Особенностью течения злокачественной инсулиномы является наличие синхронных метастазов и высокая вероятность развития тяжелых гипогликемий, в том числе рефрактерных к проводимой консервативной терапии, вследствие автономной продукции инсулина и проинсулина опухолевой массой. К наиболее частым локализациям метастазирования опухоли относятся печень (70\%) и лимфатические узлы (50\%) [3]. В редких случаях зло- качественный характер опухоли подтверждается спустя 3-9 лет после резекции первичного очага, в связи с рецидивом гипогликемического синдрома на фоне появления метахронных метастазов [1, 2].

«Золотым стандартом» диагностики инсулиномы, как и органического гиперинсулинизма в целом, является проведение 72 часовой пробы с голоданием с развитием классической триады Уиппла (появление клинической картины гипогликемии, снижение уровня глюкозы крови $\leq 2,2$ ммоль/л, купирование гипогликемического приступа внутривенным введением раствора глюкозы) и оценкой уровней инсулина, проинсулина и С-пептида крови на пике гипогликемии. Вторым этапом диагностических мероприятий является топическая диагностика опухоли [3].

В этой статье мы представляем клинический случай, демонстрирующий успешное лечение пациента с низким функциональным статусом (по шкале ECOG 3) на фоне злокачественной инсулиномы с манифестацией рефрактерного гипогликемического синдрома после проведения билиопанкреатического шунтирования с продольной резекцией желудка в раннем послеоперационном периоде [4]. 
Таблица 1. Результаты лабораторных и инструментальных исследований на догоспитальном этапе

\begin{tabular}{lcc}
\hline \multicolumn{3}{c}{$\begin{array}{c}\text { Результаты биохимического и гормонального } \\
\text { исследования крови; февраль-март 2017г; }\end{array}$} \\
\hline Показатель & $\begin{array}{c}\text { Значение, единица } \\
\text { измерения }\end{array}$ & $\begin{array}{c}\text { Референсный } \\
\text { интервал }\end{array}$ \\
\hline Глюкоза & 2,26 ммоль/л & $(3,5-5,5)$ \\
Инсулин & 33,8 мкЕд/мл & $(2,1-29)$ \\
С-пептид & 4,87 нг/мл & $(0,9-7,1)$ \\
\hline
\end{tabular}

\section{ОПИСАНИЕ КЛИНИЧЕСКОГО СЛУЧАЯ}

Пациент 53 лет в апреле 2017 г. поступил в клинику эндокринологии Первого МГМУ им. И.М. Сеченова с жалобами на выраженную слабость, онемение пальцев рук, снижение памяти, эпизоды снижения гликемии до 1,8 ммоль/л, сопровождающимися дрожью и ухудшением зрения; повышение температуры тела до $38^{\circ} \mathrm{C}$, кашель, кровохарканье.

Анамнез заболевания:

У пациента с длительным стажем морбидного ожирения в возрасте 46 лет в 2010 г диагностирован сахарный диабет (ИМТ 42 кг/м²), назначен Глимепирид 2 мг/сут. В связи с частыми гипогликемиями препарат отменен спустя 2 мес, показатели гликемии при самоконтроле оставались в пределах целевых значений.

В период с 2013 г. по 2017 г. зарегистрировано четыре эпизода гипогликемии, проявлявшихся слабостью, дрожью в теле, головной болью, нарушением зрения, чувством голода, дезориентацией в пространстве, возникающие после длительных перерывов между приемами пищи. На фоне повышения аппетита отмечено увеличение массы тела на 25 кг за 4 года (ИМТ 56 кг/м²).

Неоднократные попытки консервативной коррекции массы тела были неэффективны. В феврале 2017 г пациенту выполнено билиопанкреатическое шунтирование с продольной резекцией желудка в модификации HessMarceau, а также холецистэктомия (в связи с наличием калькулезного холецистита). В раннем послеоперационном периоде появились частые рефрактерные гипогликемические состояния до 1,8 ммоль/л.

При обследовании по результатам МСКТ брюшной полости (март 2017 г.) в головке поджелудочной железы выявлен конгломерат узловых образований с высокой нативной плотностью (33-40 HU), накапливающий контрастный препарат (115 HU в артериальную фазу (69 HU). По результатам лабораторных исследований зарегистрирована гипогликемия, гиперинсулинемия (см. таблицу 1).

За период февраль-апрель 2017 г. отметил снижение массы тела на 25 кг.

Сопутствующие заболевания:

С 2004 г. артериальная гипертензия с максимальными цифрами АД 160/80 мм рт ст. На фоне приема бетаксолола 20 мг/сут в комбинации с индапамидом 1,5 мг/сут отмечалась стабилизация гемодинамических показателей.

С 1994 г. - варикозная болезнь вен нижних конечностей.

В марте 2017 г. по результатам иммунологического исследования крови выявлен положительный $\mathrm{HBsAg;}$ количественный тест ДНК НBV не проводился, сведений о биохимической активности нет; терапия не назначалась.
Физикальный осмотр при поступлении

в эндокринологический стационар

Состояние тяжелое, низкий функциональный статус (по шкале ECOG 3). Положение пассивное. Рост 176 см, вес 139 кг, ИМТ 44,9 кг/м². Температура тела 36,5ㄷ. Кожные покровы смуглые, чистые, влажные. Подкожно-жировая клетчатка развита избыточно, распределена по абдоминальному типу. Обращает на себя внимание варикозное расширение вен нижних конечностей. Отеков нет. При аускультации легких: справа - дыхание везикулярное, слева - ослабленное везикулярное, хрипов нет, чДД 20 в мин. Тоны сердца приглушены, ритмичны, шумы не выслушиваются. Пульс ритмичный, удовлетворительного наполнения, 74 уд. в мин., АД 110/60 мм рт. ст. на обеих руках. Гликемия за сутки 1,6-3,2 ммоль/л.

\section{Результаты лабораторных и инструментальных}

исследований

По результатам пробы с голоданием, проведенной в первые сутки пребывания в стационаре, подтвержден органический гиперинсулинизм: классическая триада Уиппла развилась через 6 часов после начала пробы глюкоза 1,3 ммоль/л, С-пептид 1579 пмоль/л (298-2350), инсулин 18,5 мЕ/л (2,0-29,1), соотношение ИРИ/глюкоза - 0,7. При исследовании крови на неспецифические маркеры нейроэндокринных опухолей: Хромогранин А 3,3 нмоль/л ( $\leq 2)$, NSE 9,8 нг/мл $(<17,0)$. Также по результатам проведенного обследования (клинико-лабораторные показатели) исключен синдром МЭН I типа.

При повторной оценке результатов МСКТ брюшной полости с контрастным усилением от марта 2017 г.: в крючковидном отростке поджелудочной железы визуализируется овоидной формы образование размером 22×43 мм с четкими неровными контурами, плотностью $37 \mathrm{HU}$, при контрастировании отмечается выраженное накопление KC до $90 \mathrm{HU}$ в артериальную фазу, неоднородное, в центре визуализируется участок сниженного накопления контрастного вещества; в венозную фазу до $108 \mathrm{HU}$. Данное образование интимно прилежит к задней полуокружности верхней брыжеечной вены, а также контактирует с верхней брыжеечной артерией без признаков инвазии стенки вены и артерии. Рядом в забрюшинной клетчатке визуализируются четыре выражено увеличенных лимфатических узла размером до 22 мм, шаровидной формы, неоднородной структуры, также неоднородно накапливают контрастный препарат с гиподенсным центром. Кроме этого визуализируются множественные ЛУ парааортально ниже отхождения почечных артерий, размер их до 7 мм, несколько слабее накапливают КС в отличие от вышеописанных. Правая печеночная артерия отходит от ВБА. (рис. 1, 2, 3).

На основании результатов КТ грудной клетки с контрастным усилением и уровня Д-димера (0,36 мкг/мл) была исключена тромбоэмболия легочной артерии. Выявлена внебольничная двусторонняя полисегментарная пневмония.

В биохимическом анализе крови, на фоне проводимой терапии, обращали на себя внимание: гипокалиемия 2,8-3,8 ммоль/л (3,5-5,5), гипоальбуминемия 25-32 г/л (32-48), гипопротеинемия 43,7-59 г/л (57-82); повышение печеночных трансаминаз АЛТ 118-130 ЕД/л (10-49), АСТ 58-60 ЕД/л (0-34). В общем анализе крови - ускорение СОЭ до 63 мм/ч. По результатам иммунологического 


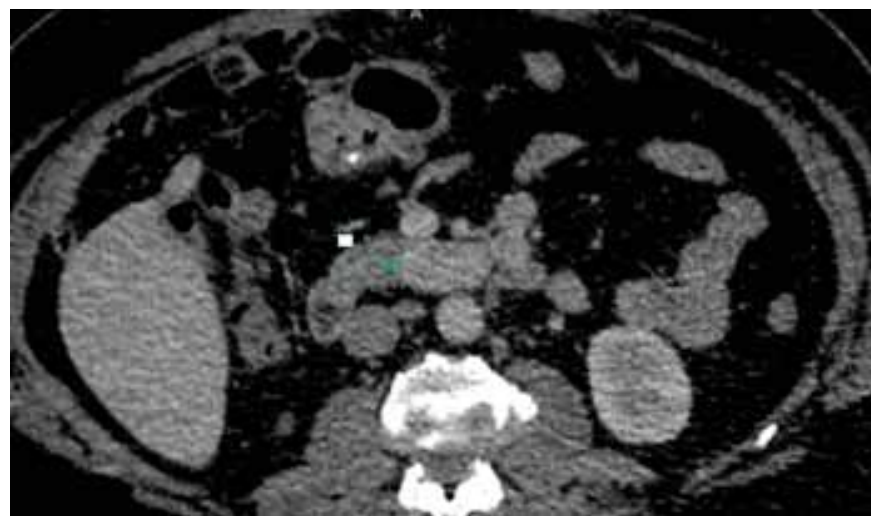

Рис. 1. МСКТ органов брюшной полости с КУ. Изоваскулярное образование в крючковидном отростке поджелудочной железы, лимфоаденопатия.

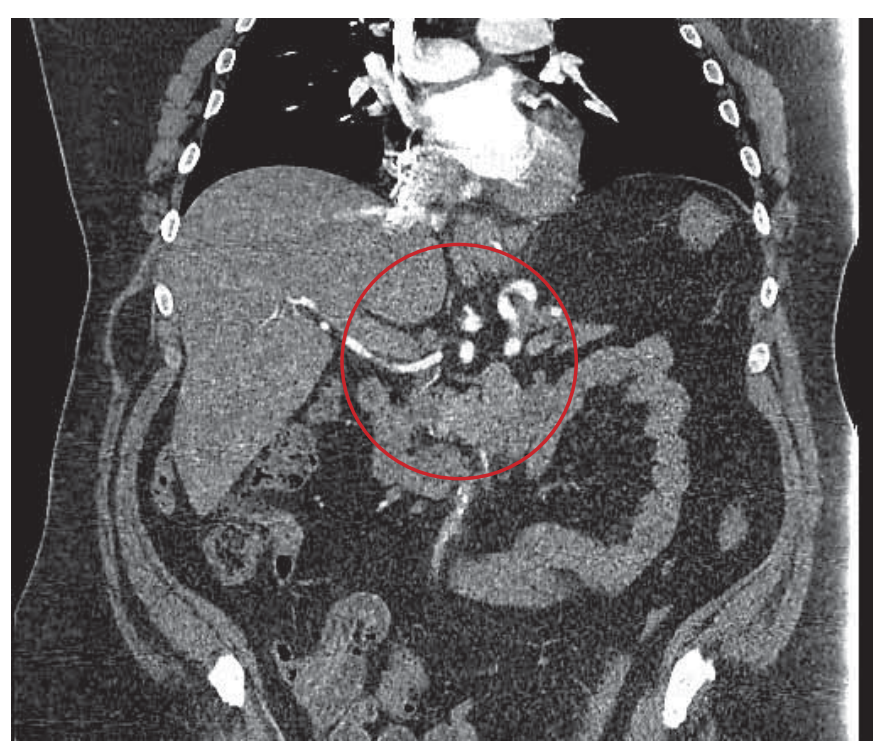

Рис. 2. МСКТ фронтальная плоскость, опухоль головки поджелудочной железы T2N1M0.

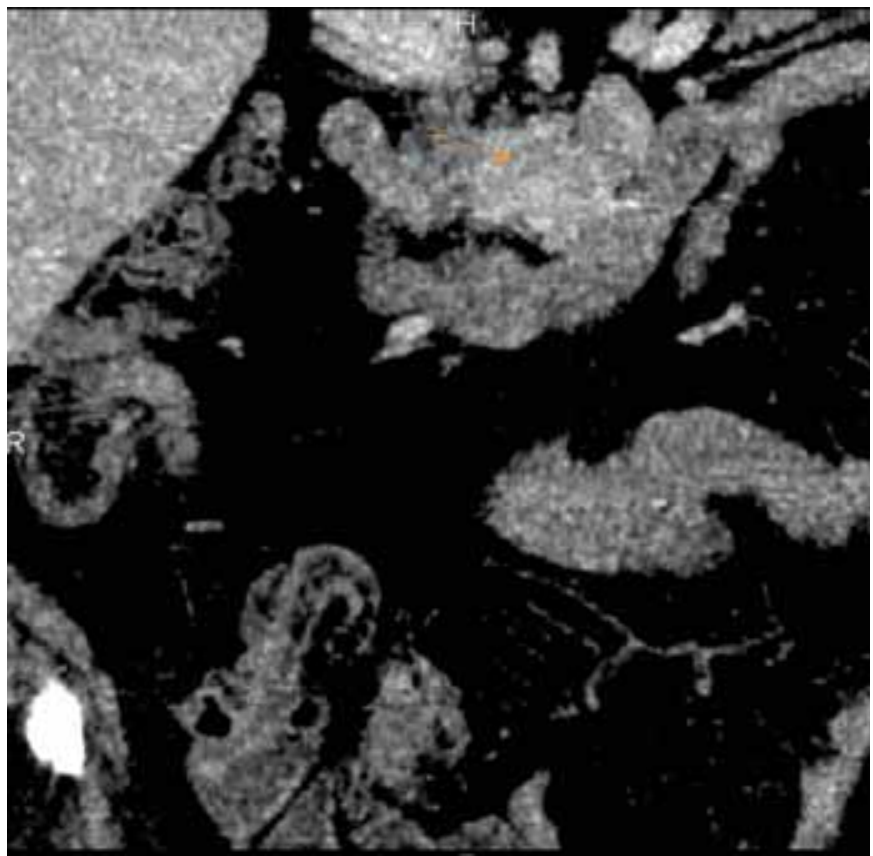

Рис. 3. МСКТ фронтальная плоскость, опухоль головки поджелудочной железы.

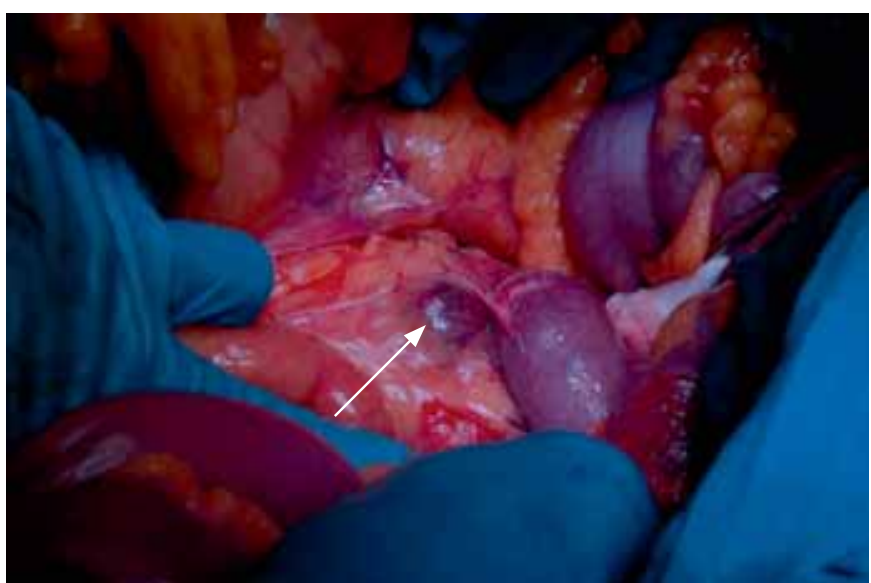

Рис. 4. Опухоль (инсулинома) головки поджелудочной железы.

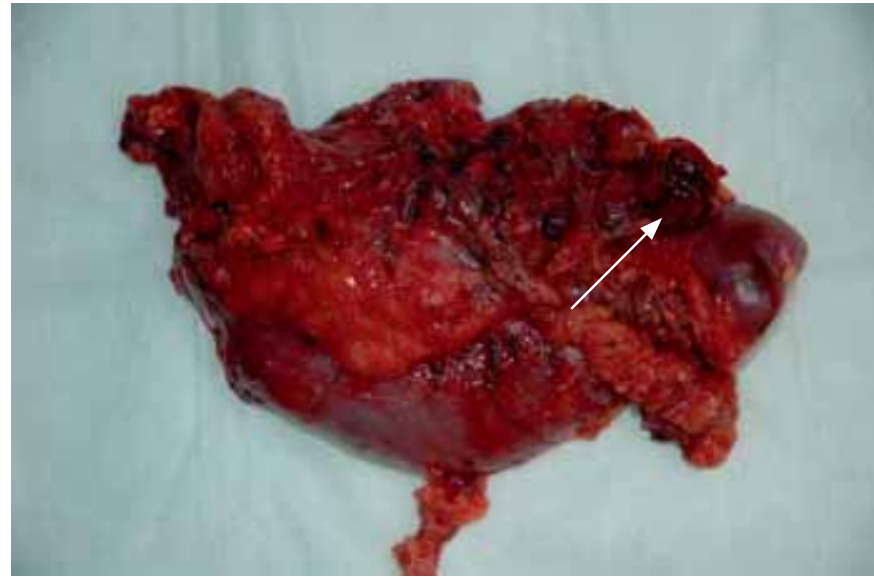

Рис. 5. Панкреатодуоденальный комплекс, лимфатические узлы.

исследования крови: повторно HBsAg - положительно; количественный тест ДНК HBV - 1,4×102 Ме/мл; маркеры вирусного гепатита D (anti-HDV, RNAHDV)- отрицательно.

\section{Лечение}

В связи с наличием частых рефрактерных гипогликемий, которые не удавалось предотвращать и купировать пероральным приемом достаточного количества углеводов, (объем желудка 100 мл), усугубляющихся наличием синдрома мальабсорбции после проведения бариатрической операции, проводилось непрерывное внутривенное введение раствора глюкозы. Ежедневно через центральный катетер внутривенно капельно вводилось 3,5-4 л 10\% раствора глюкозы; 100 мл 40\% раствора глюкозы; парентерально внутримышечно вводились глюкокортикоидные препараты: гидрокортизон 100 мг/сут с последующей заменой на преднизолон 60 мг/сут (в связи с усугублением гипокалиемии); проводилась терапия аналогами соматостатина короткого действия - октреотид 600 мкг/сут. На этом фоне гликемия в течение суток сохранялась в пределах 1,3-3,5 ммоль/л, с развитием тяжелых гипогликемий при прекращении внутривенной инфузии раствора глюкозы.

Проводилась коррекция гипокалиемии, гипопротеинемии и гипоальбуминемии с использованием раствора $\mathrm{KCl}$, сбалансированных белковых смесей для энтерального питания.

С учетом наличия двусторонней полисегментарной пневмонии проводился курс антибактериальной 
Таблица 2. Результаты лабораторных и инструментальных исследований в отдаленном послеоперационном обследовании

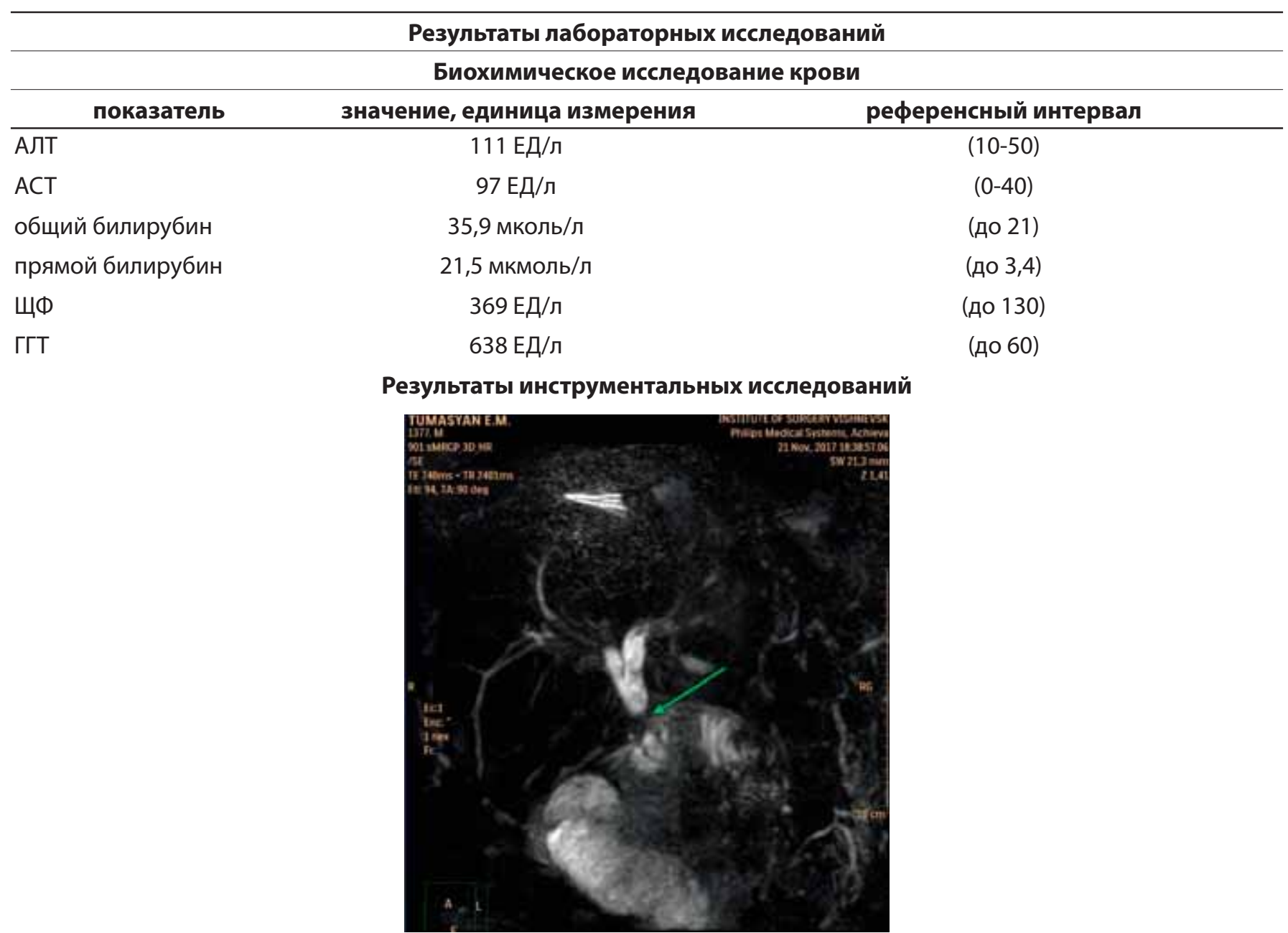

Рис. 6. Магнитно-резонансная холангиопанкреатикография, признаки стриктуры гепатикоеюноанастомоза (указано зеленой стрелкой)

(цефтриаксон 1 г/сут в/в капельно, амикацин 1 г/сут в/в капельно) и антикоагулянтной (эноксапарин натрия 1,6 мл/сут подкожно или 16000 анти-Ха МЕ), с положительным эффектом.

Несмотря на тяжесть состояния пациента, определявшую неблагоприятный прогноз, ввиду высокого операционно-анестезиологического риска и высоких рисков летального исхода в послеоперационном периоде, в мае 2017 г. выполнена расширенная панкреатодуоденальная резекция с лимфодиссекцией (рис. 4, 5). Послеоперационный период был осложнен развитием кровотечения из острой язвы гастроэнтероанастомоза (ЭГДС до проведения оперативного вмешательства не выполнялась), по поводу чего проведен эндоскопический гемостаз.

По результатам гистологического исследования удаленного панкреатодуоденального комплекса: опухоль в крючковидном отростке имеет строение нейроэндокринного рака; единичный метастаз опухоли в парапанкреатический лимфатический узел.

По данным иммуногистохимического исследования: в клетках опухоли поджелудочной железы выявлена экспрессия хромогранина А, синаптофизина, выраженная мембранная экспрессия рецепторов к соматостатину 2 типа (3+) и 5 типа (3+); экспрессия СК19 отрицательная в клетках опухоли; при исследовании с диагностическим антителом Кі-67, индекс пролиферации опухолевых кле- ток составил 1\%. Иммуноморфологические признаки опухоли соответствовали высокодифференцированной нейроэндокринной опухоли низкой степени злокачественности G1, pT2N1Mx.

\section{Исход и результаты последующего наблюдения}

В послеоперационном периоде эпизодов гипогликемий не регистрировалось, гликемия при контроле 4,0-5,4 ммоль/л, гликированный гемоглобин 4,9\%. Данных за метастатический процесс нет.

В ноябре 2017 г. при обследовании по поводу рецидивирующих жалоб на слабость, тошноту, потемнение мочи, иктеричность склер, повышения температуры тела до 38 С, диагностирована стриктура гепатикоеюноанастомоза (результаты лабораторных и инструментальных исследований представлены в таблице 2, рис. 6). Выполнено чрескожное чреспеченочное наружно-внутреннее дренирование внутрипеченочных желчных протоков под контролем ультразвуковой визуализации, баллонная дилатация зоны стриктуры.

В декабре 2017 г., в связи с рецидивирующей гемобилией, наружно-внутренний транспеченочный дренаж переведен в наружный, на этом фоне состояние пациента оставалось стабильным. В январе 2018 г. наружный транспеченочный дренаж вновь переведен в наружно-внутренний, осложнений не отмечено. В апреле 
Таблица 3. Дифференциальная диагностика гипогликемий после бариатрических операций (гастрошунтирования), Malik S. et al.

\begin{tabular}{|c|c|c|c|}
\hline & $\begin{array}{l}\text { поздний демпинг- } \\
\text { синдром }\end{array}$ & $\begin{array}{c}\text { неинсулиномный } \\
\text { панкреатогенный } \\
\text { гипогликемический синдром/ } \\
\text { незидиобластоз }\end{array}$ & инсулинома \\
\hline гипогликемии после еды & + & + & + \\
\hline гипогликемии натощак & - & - & + \\
\hline $\begin{array}{l}\text { уровень ИРИ плазмы крови } \\
\text { натощак }\end{array}$ & норма & норма & повышен \\
\hline $\begin{array}{l}\text { уровень C-пептида плазмы крови } \\
\text { натощак }\end{array}$ & норма & норма & повышен \\
\hline $\begin{array}{l}\text { уровни ИРИ, С-пептида, } \\
\text { проинсулина на фоне гипогликемии }\end{array}$ & повышены & повышены & повышены \\
\hline 72 ч проба с голоданием & - & - & + \\
\hline $\begin{array}{l}\text { стимулированный глюконатом } \\
\text { кальция забор артериальной крови }\end{array}$ & $\begin{array}{c}\text { отрицательно } \\
\text { или диффузная } \\
\text { секреция инсулина }\end{array}$ & $\begin{array}{c}\text { диффузная гиперсекреция } \\
\text { инсулина }\end{array}$ & $\begin{array}{c}\text { локализованная } \\
\text { гиперсекреция } \\
\text { инсулина }\end{array}$ \\
\hline $\begin{array}{l}\text { визуализирующие } \\
\text { инструментальные методы } \\
\text { исследования поджелудочной } \\
\text { железы }\end{array}$ & отрицательно & отрицательно & $\begin{array}{c}\text { положительно } \\
\text { (опухоль } \\
\text { поджелудочной } \\
\text { железы) }\end{array}$ \\
\hline Лечение & $\begin{array}{c}\text { - соблюдение } \\
\text { рекомендаций } \\
\text { по диете } \\
\text { - медикаментозная } \\
\text { терапия } \\
\text { - хирургическое } \\
\text { лечение }\end{array}$ & $\begin{array}{c}\text { - уменьшение симптомов } \\
\text { (от половины до значительного } \\
\text { уровня) на фоне соблюдения } \\
\text { рекомендаций по питанию } \\
\text { - медикаментозная терапия } \\
\text { - хирургическое лечение }\end{array}$ & $\begin{array}{l}\text { хирургическое } \\
\text { лечение }\end{array}$ \\
\hline
\end{tabular}

2018 г. - повторная баллонная дилатация билиодигистивного анастомоза. Состояние остается стабильным.

\section{ОБСУЖДЕНИЕ}

Ожирение в современном мире приобретает масштабы глобальной эпидемии и является фактором риска развития сердечно-сосудистых заболеваний, нарушений углеводного обмена, поражения опорно-двигательного аппарата, развития неалкогольной жировой болезни печени и синдрома обструктивного апноэ сна.

В силу низкой долгосрочной эффективности консервативной терапии у пациентов с морбидным ожирением, бариатрическая хирургия оказывается наиболее эффективным вариантом лечения, который приводит к значительному снижению веса в среднем на 30-40 кг (на $60 \%$ от исходных показателей). В течение последних 10 лет в литературе все чаще встречаются данные о появлении гипогликемических состояний у пациентов после бариатрических операций. Основной причиной которых является поздний демпинг-синдром, развивающийся в следствии реактивной гиперинсулинемии на фоне усиленного «инкретинового эффекта»; более редкой причиной может быть органический гиперинсулинизм (незидиобластоз, инсулинома) [5].

Первое упоминание в литературе о развитии органического гиперинсулинизма у пациентов после бариатрических операций встречается в 2005 г. в работах сотрудников клиники Мейо. Авторы указывают на шесть случаев развития гипогликемий после бариатрических операций, пять из которых, впоследствии развивались в результате незидиобластоза, и только в одном случае причиной гипогликемического синдрома была инсулинома. [6].

Malik S. и соавт. сформулировали основные принципы дифференциальной диагностики гипогликемических состояний, развивающихся после проведения бариатрических операций, результаты представлены в таблице 3 [5].

В представленном нами клиническом случае у пациента единичные эпизоды гипогликемий регистрировались еще до проведения бариатрической операции, однако не нашли должного внимания со стороны медицинского персонала, что свидетельствует о низкой осведомленности врачей других специальностей о возможных причинах гипогликемий и необходимости проведения специфического обследования (проба с 72 часовым голоданием) у пациентов с гипогликемическим синдромом. Частые вынужденные приемы пищи, в том числе ночная еда были расценены, как расстройство пищевого поведения (компульсивная еда). В то время как наиболее вероятной причиной стремительного увеличения массы тела пациента являлась инсулинпродуцирующая нейроэндокринная опухоль поджелудочной железы.

Первый подобный случай в литературе был представлен L. Zagury и соавторами, которые сообщили о женщине 65 лет с морбидным ожирением (ИМТ 48,8 кг/м²), у которой за год до проведения бариатрической операции зарегистрирована прибавка в весе на 16 кг, несмо- 
тря на прием медикаментозной терапии, направленной на снижение массы тела. Ввиду отсутствия эффекта от медикаментозной терапии, невозможности соблюдения рекомендаций по питанию, пациентке выполнено желудочное шунтирование Fobi-Capella. B послеоперационном периоде появились жалобы на учащенное сердцебиение, тошноту, рвоту, расцененные как демпинг-синдром. При обследовании подтвержден органический гиперинсулинизм, обнаружена инсулинома, проведено оперативное лечение [7].

Еще один случай инсулиномы, приведший к развитию морбидного ожирения описан Seshadri P и соавторами. У женщины 61 года с длительным стажем морбидного ожирения (вес 131 кг) за четыре года до бариатрической операции появились жалобы на потливость и слабость после еды, которые были расценены как развивающийся сахарный диабет, с учетом отягощенного наследственного анамнеза. Выполнено лапароскопическое бандажирование желудка. В течение четырех месяцев после выполненной хирургической процедуры отметила снижение веса на 10 кг, симптомы гипогликемий, которым не предала должного значения. В последующем эпизоды слабости и усталости стали более частыми и прекращались после приема легкоусваиваемых углеводов (при самоконтроле уровень глюкозы 1,65-3,86 ммоль/л); за три месяца прибавила в весе 6 кг. По результатам пробы с голоданием подтверждена гиперинсулинемическая гипогликемия, по данным инструментальных исследований выявлена опухоль головки поджелудочной железы, проведена энуклеация опухоли. После операции отмечено снижение массы тела на 8 кг, эпизодов гипогликемий не возникало [8].

Об органической причине ожирения может свидетельствовать наличие в анамнезе симптомов гипогликемий; продолжающаяся прибавка веса, несмотря на консервативную терапию ожирения; зарегистрированные гипогликемии в ходе орального глюкозотолерантного теста могут в свою очередь свидетельствовать об автономной продукции инсулина. Изолированное повышение уровней инсулина и С-пептида плазмы крови не должны являться верифицирующим диагностическим тестом органического гиперинсулинизма у пациентов с морбидным ожирением, так как достаточно часто встречаются в данной когорте пациентов и связаны с наличием инсулинорезистентности. При подозрении на инсулиному «золотым стандартом» диагностики остается проведение 72 часовой пробы с голоданием.

Развитие органического гиперинсулинизма может встречаться у пациентов спустя несколько месяцев и даже лет после проведения бариатрических вмешательств. Так в 2008 г. Abellan и соавторы описали развитие нейрогликопенической симптоматики натощак у пациента 51 года с морбидным ожирением (ИМТ 55,8 кг/м²) через 3 месяца после выполнения вертикальной гастропластики и кишечного шунтирования, сопровождающейся снижением гликемии до 2 ммоль/л. В дальнейшем отмечено увеличение частота эпизодов гипогликемий, появились эпизоды потери сознания и судорог. По данным обследования подтвержден органический гиперинсулинизм, при топической диагностике выявлена опухоль в хвосте поджелудочной железы. Выполнена дистальная резекция поджелудочной железы, по результатам гистологического исследования подтверждена ин- сулинома с явлениями незидиобластоза в окружающей ткани поджелудочной железы [9]. J. Duff и соавторы сообщили о развитии инсулиномы у пациента через 7 лет после выполнения гастрошунтирования с межкишечным анастомозом по Ру и подчеркнули необходимость использования наиболее информативных и специфичных инструментальных исследований для топической диагностики [10].

Кроме того, в литературе описаны случаи длительного и безуспешного лечения пациентов с поздним демпинг-синдромом после хирургических вмешательств на верхних отделах желудочно-кишечного тракта по поводу язвенной болезни желудка, двенадцатиперстной кишки, рака желудка (стволовая ваготомия с пилоропластикой, резекция антрального отдела желудка с последующей гастроеюностомией, гастрэктомия и пр.), которые при более детальном обследовании оказались органическим гиперинсулинизмом (инсулиномой) [12-14].

Суммируя вышесказанное, можно заключить, что демпинг-синдром является частой, но не единственной причиной гипогликемических состояний, развивающихся у пациентов после выполнения бариатрических операций. Недостаточная оценка причин гипогликемических состояний у данной группы пациентов и несвоевременная диагностика органического гиперинсулинизма могут представлять серьезную опасность для жизни пациента, приводя к инвалидизации или смертельному исходу.

\section{ЗАКЛЮЧЕНИЕ}

Продемонстрированный в данной статье случай отображает особенности клинического течения рефрактерного гипогликемического синдрома у пациента со злокачественной инсулиномой, усугубляющегося вследствие развития синдрома мальабсорбции после шунтирующей операции; основные этапы диагностики и трудности, возникшие в ходе лечения ввиду тяжелого соматического состояния пациента. Возможным дополнительным фактором рефрактерности к медикаментозной терапии гипогликемии в данном случае являлось наличие у пациента заболевания печени - гепатит смешанной этиологии (вирусный гепатит В, неалкогольная жировая болезнь печени), что косвенно подтверждает отсутствие эффекта от введения преднизолона.

Перед проведением бариатрических операций по поводу морбидного ожирения целесообразным является расспрос пациента о наличии симптомов гипогликемий в анамнезе.

Расстройства приема пищи на фоне ожирения (компульсивная еда) могут маскировать нейрогликопеническую симптоматику инсулином, затрудняя своевременную диагностику.

\section{ДОПОЛНИТЕЛЬНАЯ ИНФОРМАЦИЯ}

Согласие пациента. Пациент добровольно подписал информированное согласие на публикацию персональной медицинской информации в обезличенной форме в журнале «Ожирение и метаболизм».

Конфликт интересов. Авторы декларируют отсутствие явных и потенциальных конфликтов интересов, связанных с публикацией настоящей статьи. 


\section{СПИСОК ЛИТЕРАТУРЫ | REFERENCES}

1. Baudin E, Caron P, Lombard-Bohas C, et al. Malignant insulinoma: Recommendations for characterisation and treatment. Ann Endocrinol (Paris). 2013. doi: 10.1016/j.ando.2013.07.001

2. Maresova D, Kozler P, Pokorny J. Neuronal excitability changes depend on the time course of cellular edema induced by water intoxication in young rats. Neuro Endocrinol Lett. 2016;37(3):207-212. PMID: 27618603

3. Hirshberg B, Cochran C, Skarulis MC, et al. Malignant insulinoma. Cancer. 2005;104(2):264-272. doi: 10.1002/cncr.21179

4. Oken MM, Creech RH, Tormey DC, et al. Toxicity and response criteria of the Eastern Cooperative Oncology Group. Am J Clin Oncol. 1982;5(6):649-656. doi: 10.1097/00000421-198212000-00014

5. Malik S, Mitchell JE, Steffen K, et al. Recognition and management of hyperinsulinemic hypoglycemia after bariatric surgery. Obes Res Clin Pract. 2016. doi: 10.1016/j.orcp.2015.07.003

6. Mulla CM, Storino A, Yee EU, et al. Insulinoma After Bariatric Surgery: Diagnostic Dilemma and Therapeutic Approaches. Obes Surg. 2016;26(4):874-881. doi: 10.1007/s11695-016-2092-5

7. Zagury L, Moreira RO, Guedes EP, et al. Insulinoma Misdiagnosed as Dumping Syndrome after Bariatric Surgery. Obes Surg. 2004;14(1):120-123. doi: 10.1381/096089204772787419

8. Seshadri P, Lenhard MJ, Bennett J, et al. Rare case of insulinoma diagnosed after laparoscopic gas- tric banding. Surg Obes Relat Dis. 2009;5(1):123-127. doi: 10.1016/j.soard.2008.03.250

9. Abellán P, Cámara R, Merino-Torres JF, et al. Severe hypoglycemia after gastric bypass surgery for morbid obesity. Diabetes Res Clin Pract. 2008. doi: 10.1016/j.diabres.2007.07.017

10. Duff JM, Ghayee HK, Weber M, Thomas RM. Delayed Imaging Presentation of a Symptomatic Insulinoma After Bariatric Surgery. J Gastrointest Surg. 2017;21(2):412-414. doi: 10.1007/s11605-016-3219-7

11. Qian SY, Hare MJL, Pham A, Topliss DJ. Insulinoma presenting with post-prandial hypoglycaemia following fundoplication. Endocrinol Diabetes Metab Case Reports. 2018;(1). doi: 10.1530/EDM-17-0131

12. Koca YS, Aydın B, Koca T, Bülbül MT, Tamer MN. Insulinoma-Induced Hypoglycemia in a Patient with Insulinoma after Gastrojejunostomy for Prepyloric Ulcer. Case Rep Surg. 2015;2015:1-3. doi: 10.1155/2015/127914

13. Iglesias P, Ais G, Pastrana M, Etreros J, Salinas S, Diez JJ. Insulinoma-Induced hypoglycemia in a patient with nesidiodysplasia after vagomotomy and pyloroplasty for duodenal ulcer. Am J Med Sci. 2009. doi: 10.1097/MAJ.0b013e3181907b06

14. Sato T, Narita T, Hosoba M, et al. A case of insulinoma following total gastrectomy-Effects of an al pha-glucosidase inhibitor on suppressing GIP and GLP-1 elevations. Diabetes Res Clin Pract. 2010;88(1):e4-e6. doi: 10.1016/j.diabres.2010.01.006

\section{ИНФОРМАЦИЯ ОБ АВТОРАХ [AUTHORS INFO]}

* Малолеткина Екатерина Станиславовна [Ekaterina S. Maloletkina, MD];

ORCID: https://orcid.org/0000-0001-9881-5459; eLibrary SPIN: 6794-2220; e-mail: Maloletkinae@mail.ru

Гурова Олеся Юрьевна, к.м.н. [Olesya Yu. Gurova, MD, PhD]; ORCID: https://orcid.org/0000-0003-2576-4421; eLibrary SPIN: 1257-5499; e-mail: gurova79@inbox.ru

Фадеев Валентин Викторович, д.м.н., профессор, член-корреспондент PAH [Valentin V. Fadeev, MD, ScD, professor, corresponding member of the RAS]; ORCID: https://orcid.org/0000-0002-3026-6315; eLibrary SPIN: 6825-8417;

e-mail: walfad@mail.ru

Егоров Вячеслав Иванович. д.м.Н., профессор [Vyacheslav I. Egorov, MD, ScD, professor];

ORCID: https://orcid.org/0000-0002-8805-7604; eLibrary SPIN: 4487-1663; e-mail: egorov12333@gmail.com

Петров Роман Викторович, к.M.H. [Petrov V. Roman, MD, PhD]; ORCID: https://orcid.org/0000-0003-3872-7363;

e-mail: petrov-r-v@yandex.ru

Бельцевич Дмитрий Германович, д.м.н., профессор [Beltsevich G. Dmitry MD, ScD, professor];

ORCID: https://orcid.org/0000-0001-7098-4584; eLibrary SPIN: 4475-6327; e-mail: beltsevich@rambler.ru

Воробьев Андрей Васильевич [Vorob'ev V. Andrei, MD]; ORCID: https://orcid.org/0000-0002-2679-289X;

e-mail:podolsk@gmail.com

\section{ЦИТИРОВАТЬ:}

Малолеткина Е.С., Гурова О.Ю., Фадеев В.В., Егоров В.И., Петров Р.В., Бельцевич ДГ., Воробьев А.В. Случай инсулиномы, выявленной у пациента после бариатрической операциипо поводу морбидного ожирения // Ожирение и метаболизм. - 2019. — Т.16. — №1. — С. 74-80. doi: 10.14341/omet9751

\section{TO CITE THIS ARTICLE:}

Maloletkina ES, Gurova OYu, Fadeev VV, Egorov VI, Petrov RV, Beltsevich DG, Vorob'ev AV. Case of insulinoma detected in a patient after bariatric operation for morbid obesity. Obesity and metabolism. 2019;16(1):74-80. doi: 10.14341/omet9751 